\title{
Experimental study on a nonlinear vibration isolator based on a post-buckled inverted L-shaped beam
}

\author{
Takumi SASAKI*, Timothy WATERS** and Yumiko TSUJI* \\ *Department of Environmental Engineering, University of Kitakyushu \\ 1-1 Hibikino, Wakamatsu-ku, Kitakyushu, Fukuoka 808-0135, Japan \\ E-mail: sasa@kitakyu-u.ac.jp \\ ${ }^{* *}$ Institute of Sound and Vibration Research, University of Southampton \\ Highfield, Southampton SO17 1BJ, United Kingdom
}

Received 8 January 2015

\begin{abstract}
In this paper, the characteristics and the effectiveness of a nonlinear passive vibration isolator based on a post-buckled beam is investigated experimentally. The intended application is specifically isolation in the vertical direction where the isolator is required to be sufficiently stiff statically to bear the weight of the isolated mass. The isolator consists of two beams joined to form an inverted L-shape and the weight of the isolated mass is taken to act at the vertex. If the weight of isolated mass is larger than the buckling load of the L-shaped beam then the beam buckles in one of two modes, one of which is unstable. In this paper, the static restoring force of the unstable mode is measured and an appropriately selected coil spring is added to counteract the negative stiffness of the beam. The resulting system presents a dramatically lower stiffness to small excursions about its equilibrium position in its buckled state but maintains its static load bearing capability. Free vibration measurements are presented which show some amplitude dependency of the natural frequency for large amplitude motion. Low amplitude harmonic base excitation measurements are also conducted from which transmissibility measurements are obtained and compared with corresponding results from a Finite Element model. The fundamental resonance is about $80 \%$ lower than that achievable by a comparable linear isolator. However the potential improvement in isolation performance has not been fully realised in the prototype design due to the presence of higher frequency internal resonances of the isolator, mitigation of which is the focus of ongoing work.
\end{abstract}

Key words : Base isolation, Nonlinear isolator, Free vibration, Transmissibility, Quasi-Zero-Stiffness (QZS)

\section{Introduction}

Passive isolators remain the most commonly adopted form of isolator due to their simplicity, stability and low cost. Their effectiveness is reliant on being able to achieve a low natural frequency when supporting an isolated object. However, when applied to isolation in the vertical direction, low stiffness can result in impractically large static deflection due to the weight of the isolated object. These conflicting requirements can in principle be reconciled by adopting a nonlinear isolator that exhibits high stiffness when loaded statically and low stiffness in relation to small oscillations about the static equilibrium position. A device with such a nonlinear characteristic can be realized by inserting in parallel with the main load bearing isolator element a snap-through mechanism with negative stiffness. Systems that are tuned to counteract the positive stiffness almost entirely are commonly referred to as Quasi-Zero Stiffness (QZS) devices. Examples in the literature include spring lever mechanisms (Park and Luu, 2007, Carrella, 2010, Xingtian, et al., 2013, Le and Ahn, 2011, Araki, et al., 2013), bi-stable plates (Shaw, et al., 2013) and magnets (Robertson, et al., 2013, Carrella, et al., 2008). An alternative approach is to make use of a post-buckled element that maintains much of its static stiffness in its buckled state but whose tangent stiffness dramatically reduces (Winterflood, et al., 2002, Abolfathi, et al., 2013). A comprehensive review of nonlinear isolators can be found in the review of Ibrahim, 2008. In practice, experimental rigs based on either of these principles can be complex and cumbersome to 
accommodate hinged conditions and/or to constrain motion in the non-vertical directions.

The motivation for this paper is the pursuit of a simple, compact configuration of nonlinear spring element. An inverted L-shaped beam has been chosen for this investigation, although similar truss-like isolators are conceivable. The weight of the isolated mass is taken to act at the vertex causing the vertical member to buckle when the weight is sufficiently large. In its stable buckling mode the gradient of the force-deflection curve at the equilibrium position (called "tangent stiffness" hereafter) is considerably smaller than in its pre-buckled state. The particular focus of this paper, however, is the unstable buckling mode which requires an additional spring to restore stability but can be tuned, in principle, to achieve QZS.

The system is described in section 2 and its measured static behaviour is reported in section 3 . A free vibration measurement of the mass-loaded isolator is reported in section 4 which confirms the potential of such an isolator for both achieving a low fundamental natural frequency and exhibiting nonlinear dynamic response. Section 5 is concerned with the measurement of vibration transmissibility for a small amplitude harmonic base input and comparison with corresponding results from a Finite Element model. The prominence of higher order modes in the transmissibility is highlighted and examined in section 6 through FE modal analysis and high speed camera imaging of the structure under test. A preliminary prediction for a revised design to address this issue is briefly shown in section 7.

\section{Description of Inverted L-shaped Beam Isolator \\ 2.1 Conceptual design}

The inverted L-shaped beam isolator consists of two beams which are joined to form a right angle, as shown in Fig. 1. One beam is orientated vertically and the other horizontally, and the other end of each beam is fixed to a common base. The weight of the isolated mass is taken to act at the vertex of the L-shaped beam. If the weight of the isolated object is larger than the buckling load of the isolator, then the L-shaped beam will buckle in one of two buckling modes shown in Fig. 2 and Fig. 3.

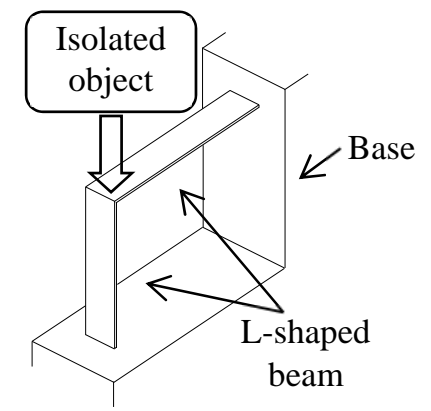

Fig. 1 A schematic of the inverted L-shaped beam isolator showing the two beams joined to form a right angle and the weight of the isolated mass acting at the vertex.

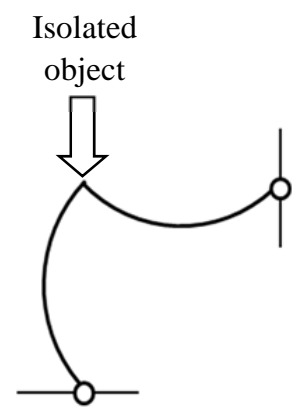

Fig. 2 The "stable buckling mode" in which the vertical beam buckles outwards and the tangent stiffness in the post-buckled state is consistently positive.

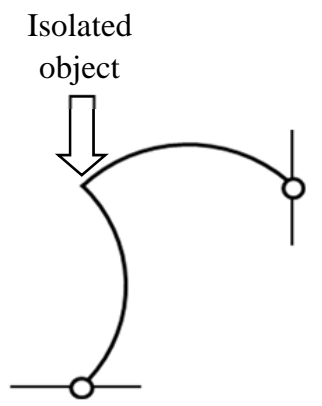

Fig. 3 The "unstable buckling mode" in which the vertical beam buckles inwards and the tangent stiffness becomes negative in the post-buckled state. 
The buckling mode of Fig.2 is referred to as the "stable buckling mode", for which the tangent stiffness in the post-buckled state is consistently positive and considerably smaller than in its pre-buckled state. The buckling mode of Fig. 3 is referred to as the "unstable buckling mode" on account of its region of negative tangent stiffness in the post-buckled state. The unstable buckling mode can be stabilized by installing in parallel a coil spring whose spring constant is larger in magnitude than the negative stiffness of the L-shaped beam. This configuration is shown within a base frame in Fig. 4. Additionally, if the spring constant of the coil spring is chosen so as only just to cancel the negative stiffness of the L-beam, then in principle QZS can be realized. It is for this potential that the unstable buckling mode is chosen here for subsequent static and dynamic analysis.

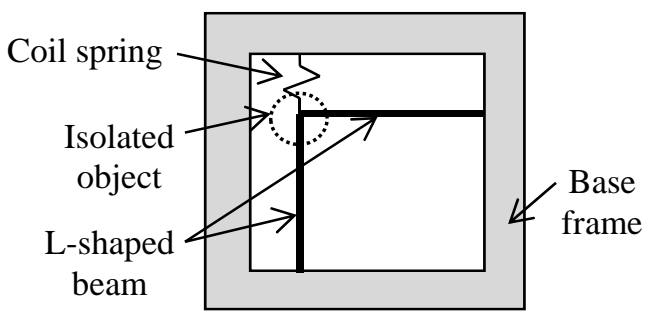

Fig. 4 A schematic of the inverted L-shaped beam isolator using its unstable buckling mode. By installing a coil spring in parallel whose spring constant is just sufficient to cancel the negative stiffness of the L-beam, the unstable buckling mode can be stabilized and very low tangent stiffness obtained.

\subsection{Experimental realisation}

Figure 5 shows a photograph of the experimental setup mounted rigidly to the table of an electrodynamic shaker. The L-shaped beam was made of blade spring (SUS 304-CSP in Japanese Industrial Standards). The two beams are welded at their join to a $5 \mathrm{~mm}$ diameter bar orientated perpendicularly to the beams which supports the isolated mass, chosen here as $1.638 \mathrm{~kg}$ to load the system to a desired post-buckled state. The base frame was built from aluminium frame of rectangular cross-section $80 \mathrm{~mm}$ x $40 \mathrm{~mm}$. A coil spring of stiffness $308 \mathrm{~N} / \mathrm{m}$ was installed between the bar and the base frame (behind the isolated object in Fig. 5). The specification of this system is summarized in table 1.

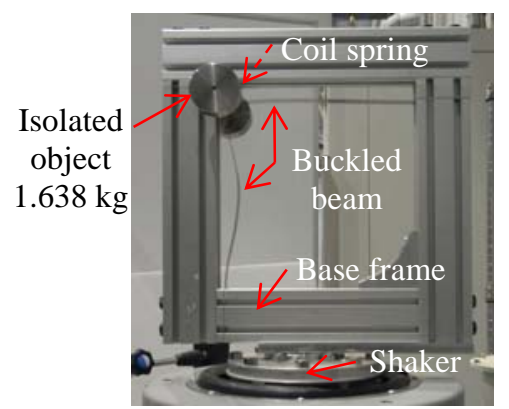

Fig. 5 Photograph of the experimental setup showing the beam mounted in the base frame and under static load. The coil spring was installed between the vertex of the beam structure and the base frame.

Table 1 Specification of the experimental setup

\begin{tabular}{|c|c|c|}
\hline \multirow{2}{*}{$\begin{array}{c}\text { Vertical } \\
\text { beam }\end{array}$} & Length [mm] & 283 \\
\cline { 2 - 3 } & Thickness [mm] & 0.5 \\
\hline $\begin{array}{c}\text { Horizontal } \\
\text { beam }\end{array}$ & Length [mm] & 283 \\
\cline { 2 - 3 } & Thickness [mm] & 0.7 \\
\cline { 2 - 3 } $\begin{array}{c}\text { Isolated } \\
\text { object }\end{array}$ & $\begin{array}{c}\text { Mass [kg] } \\
\text { Moment of inertia }\end{array}$ & $\begin{array}{c}I_{x}=3229 \times 10^{-6} \\
I_{y}=3229 \times 10^{-6}\end{array}$ \\
\hline $\begin{array}{c}\text { Coil } \\
\text { spring }\end{array}$ & $\begin{array}{c}\text { Spring constant } \\
{[\mathrm{N} / \mathrm{m}]}\end{array}$ & 308 \\
\hline
\end{tabular}

\section{Static behaviour}

The static force-deflection curve of the L-shaped beam was measured in its unstable buckling mode. In this experiment the isolated mass was not present. A load cell was set on a servo linear slider and a quasi-static vertical displacement was imposed at the vertex of the L-shaped beam. The displacement was measured using a laser displacement meter and the force was measured using a load cell. A low pass filter was applied to the force signal. The green and black lines in Fig. 6 show the measured restoring force against imposed displacement for the L-shaped beam with and without the coil spring installed. From these lines, it was confirmed that the L-shaped beam without a coil spring displays negative stiffness in its post-buckled state but that it is adequately stabilized by the positive stiffness of the coil spring. The black and red lines in Fig. 7 show the static stiffness (primary vertical axis) and the 
tangent stiffness (secondary vertical axis) for the stabilized system (green line) in Fig. 6. The static stiffness is calculated as $F(x) / x$ where $x$ is the vertical displacement and $F(x)$ is the restoring force. The tangent stiffness, $d F / d x$, was obtained from the derivative of a fit to the data in Fig. 6. Far into the buckled region ( $x \sim 8 \mathrm{~mm})$ the tangent stiffness is about one order of magnitude smaller than the static stiffness and this reduces to about $1 / 30^{\text {th }}$ at $3 \mathrm{~mm}$ deflection, for example. Even lower tangent stiffness can be achieved but the expense of less allowable travel before snapping through (at $x \sim 1 \mathrm{~mm}$ ) to the unbuckled region.

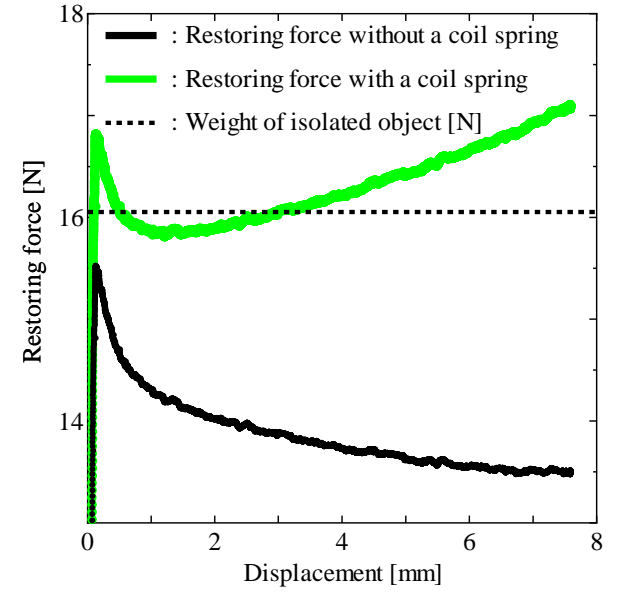

Fig. 6 Measured restoring force of the L-shaped beam without a coil spring (black) and with a coil spring (green) against imposed vertical displacement. The L-shaped beam without a coil spring displays negative stiffness in its post-buckled state and it was stabilized by installing a coil spring.

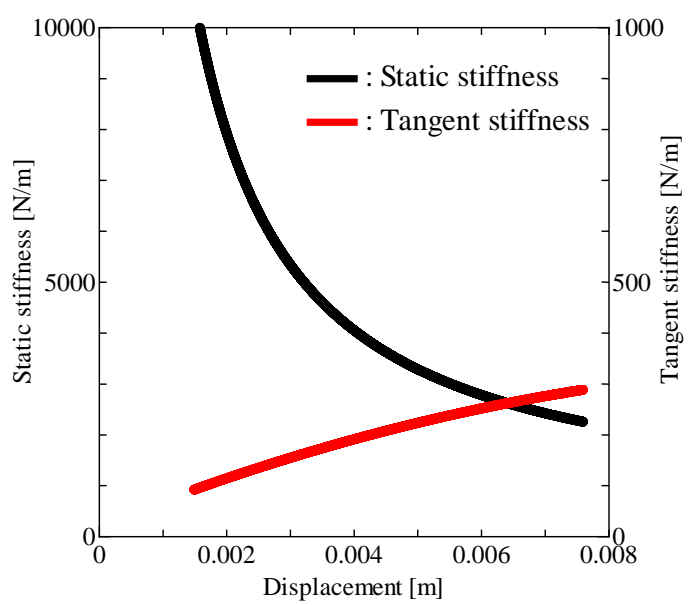

Fig. 7 The static stiffness (black, primary y-axis) and the tangent stiffness (red, secondary y-axis) calculated from a fit to the green line in Fig. 6. This system exhibits high static and low tangent stiffness.

\section{Free vibration}

A mass of $1.638 \mathrm{~kg}$ was mounted on the isolator and the beam was made to buckle inwardly causing a static deflection of about 3mm (see the dashed line in Fig. 6). The mass was given an initial displacement of about $2 \mathrm{~mm}$ from its equilibrium position which was large enough to anticipate an amplitude dependent natural frequency but without causing the system to snap through to its unbuckled state on release. The vertical displacement of the mass was measured by a laser displacement meter for 40 seconds at a sampling rate of $1 \mathrm{kHz}$. The instantaneous frequency was estimated by application of the Hilbert transform to the transient of the free decay. The Hilbert transform was performed in the frequency domain as follows,

$$
H(\omega)=-j \operatorname{sgn}(\omega) X(\omega)
$$

where $X(\omega)$ is the Fourier transform of the free vibration waveform $x(t)$ and $H(\omega)$ is its Hilbert transform in the frequency domain. The envelope of the response $e(t)$, the instantaneous phase $\theta(t)$ and the instantaneous frequency $f(t)$ are obtained from the following equations,

$$
\left.\begin{array}{l}
e(t)=\sqrt{x(t)^{2}+h(t)^{2}} \\
\theta(t)=\tan ^{-1} \frac{h(t)}{x(t)} \\
f(t)=\frac{d \theta(t)}{d t}
\end{array}\right\}
$$

where $h(t)$ is the inverse Fourier transform of $H(\omega)$. A low pass filter was applied to $x(t)$ and $h(t)$ to remove noise and response in higher order modes. The black and blue lines in Fig. 8 show the free vibration waveform and its Hilbert transform whereas the red line shows the envelope of the response. The effect of decaying amplitude on 
instantaneous frequency is shown by the black line in Fig. 9. The frequency is seen to increase as the amplitude decays, which is indicative of a nonlinear system with softening stiffness. Inspection of the force-deflection curve in Fig. 7 confirms that the system is softening for excursions in one direction (less compression) from the equilibrium position but hardening in the other direction.

Notwithstanding this significant asymmetry the system was modelled simplistically by an undamped Duffing oscillator, i.e. a single degree-of-freedom mass-spring system with linear and cubic stiffness, for which the equation of motion for free vibration is given by

$$
\ddot{x}+\omega_{n}^{2} x+\varepsilon \omega_{n}^{2} x^{3}=0
$$

The red line in Fig. 9 shows the natural frequency of a Duffing oscillator for a range of initial displacements and hence amplitudes of free vibration. The nonlinear stiffness coefficient was chosen as $\varepsilon=-1.5 \times 10^{4} \mathrm{~m}^{-2}$, which corresponds to a softening system, and the linear natural angular frequency was taken as $\omega_{n}=2 \pi \times 1.98 \mathrm{rad} / \mathrm{s}$. The curve is in reasonably good agreement with the measured instantaneous natural frequency values.

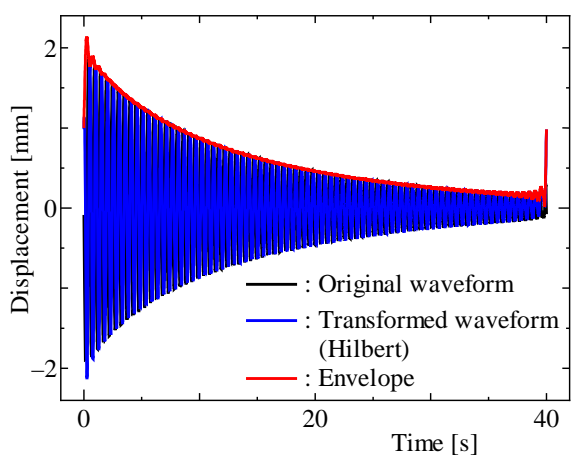

Fig. 8 Free vibration waveform (black), its Hilbert transform (blue) and the response envelope (red)

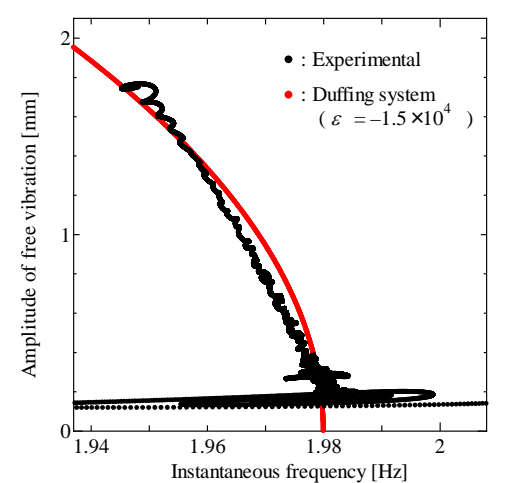

Fig. 9 The instantaneous frequency as a function of amplitude. Measured (black) and a Duffing oscillator fitted to the data (red).

\section{Transmissibility}

The isolator loaded with the $1.638 \mathrm{~kg}$ mass was mounted on an electrodynamic shaker and subjected to small amplitude harmonic base motion in order to measure the transmissibility of the linearized system. The excitation was displacement controlled at $1.5-10 \mathrm{~Hz}$ and acceleration controlled from $10-300 \mathrm{~Hz}$. The excitation amplitudes that were selected are listed in table 2 . The motions of the isolated mass and base were measured using both accelerometers and laser displacement meters. The measured acceleration and displacement signals were expanded into Fourier series with respect to the base excitation frequency and the amplitude of each order was calculated. The motion transmissibility was taken as the ratio of the first order Fourier components of the two responses. The black line in Fig.10(a) shows a concatenation of displacement transmissibility below $10 \mathrm{~Hz}$ and acceleration transmissibility above $10 \mathrm{~Hz}$.

The fundamental resonance of the system occurs at about $2 \mathrm{~Hz}$ which is consistent with the natural frequency of the system measured by free vibration. By comparison, a linear spring with the same static deflection ( 3mm) under the weight of the isolated mass would have resulted in a fundamental frequency of 9.1Hz. Fig. 10(b) illustrates the improvement in transmissibility of a linear SDOF system (with a damping ratio of 10\%) when its natural frequency is reduced from $9.1 \mathrm{~Hz}$ (red) to $2 \mathrm{~Hz}$ (black). The $13 \mathrm{~dB}$ improvement in isolation at high frequencies for this idealised system has not been realised for the L-shaped isolator due to the existence of numerous other lightly damped resonances, the lowest and most predominant of which occurs at about $7 \mathrm{~Hz}$. A modal analysis to understand the nature of these higher order modes is presented in section 6. 
Table 2 Amplitudes of harmonic base input

\begin{tabular}{|c||c|}
\hline Frequency $[\mathrm{Hz}]$ & Excitation level \\
\hline \hline $1.5-2.5$ & $0.2 \mathrm{~mm}$ (Displacement control) \\
\hline $3.0-3.5$ & $0.1 \mathrm{~mm}$ (Displacement control) \\
\hline $4.0-9.5$ & $0.05 \mathrm{~mm}$ (Displacement control) \\
\hline $10.0-300.0$ & $1.0 \mathrm{~m} / \mathrm{s}^{2}$ (Acceleration control) \\
\hline
\end{tabular}

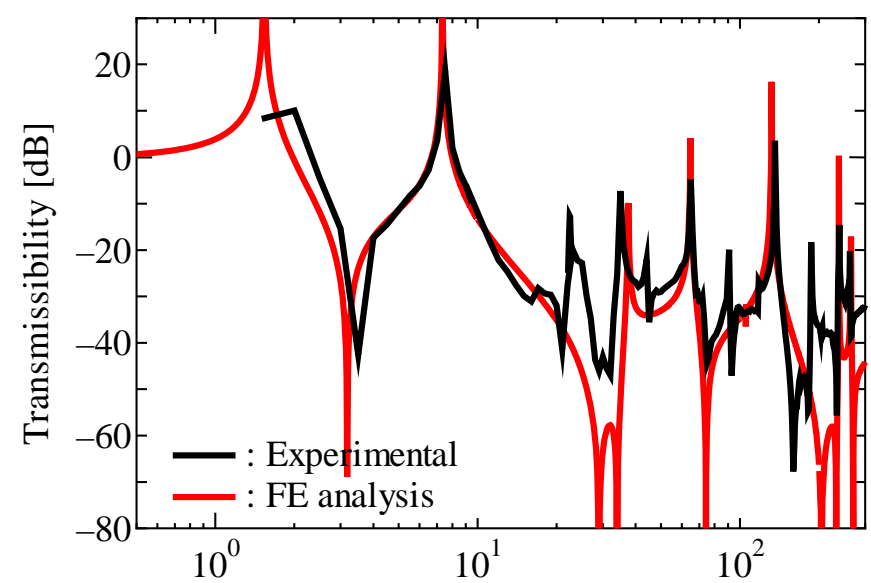

Frequency [Hz]

Fig. 10(a) The measured motion transmissibility (black) which corresponds to the 1st order component of the isolated mass divided by the 1st order component of the base. Displacement transmissibility (below $10 \mathrm{~Hz}$ ) and acceleration transmissibility (above $10 \mathrm{~Hz}$ ). The red line shows the force transmissibility obtained from a linear FE analysis in the post-buckled state.

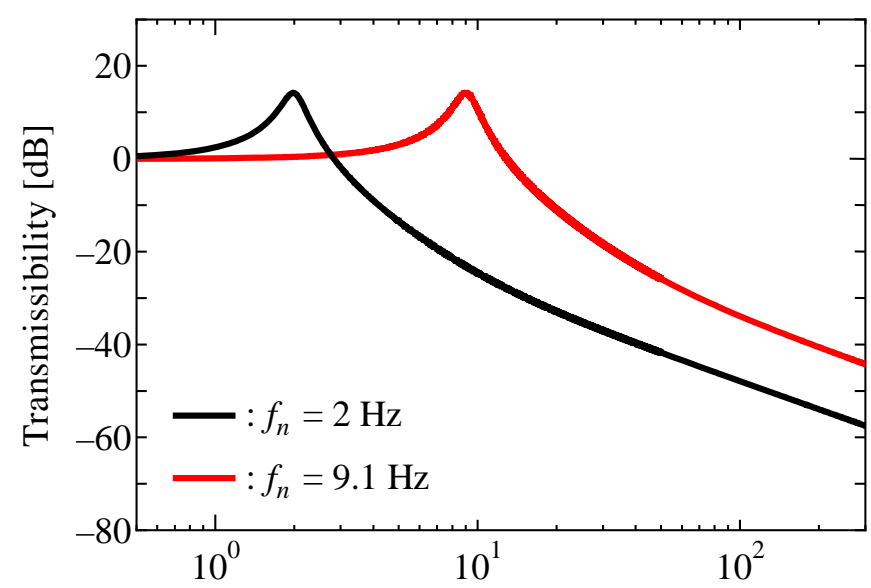

Frequency $[\mathrm{Hz}]$

Fig. 10(b) Transmissibility of linear SDOF systems with natural frequencies of $2 \mathrm{~Hz}$ (black) and 9.1Hz (red) and damping ratio of $10 \%$. The reduced natural frequency provides an additional $13 \mathrm{~dB}$ of isolation at high frequencies.

A Finite Element (FE) model was generated during the design stage and a nonlinear static analysis performed followed by modal and harmonic analyses of the linearized system in its post-buckled state (Tsuji, et al., 2014). Here, only selected results are presented for brevity. The corresponding values in table 1 and the material parameter values listed in table 3 were used for the FE model. The end of each beam is constrained with respect to translational and rotational displacement. Each beam is modelled with 100 three-dimensional quadratic finite strain beam elements. 
Table 3 Material properties for both horizontal and vertical beams

\begin{tabular}{|c|c|}
\hline Young's modulus [GPa] & 180.36 \\
\hline Poisson's ratio [-] & 0.3 \\
\hline Density $\left[\mathrm{kg} / \mathrm{m}^{3}\right]$ & 7000 \\
\hline
\end{tabular}

The transmissibility predicted by the FE model is overlaid with the experimental curve in Fig. 10(a). The frequencies of most of the additional resonances, including the predominant $7 \mathrm{~Hz}$ mode, are in good agreement and are associated with higher order modes of the isolator. Note that the amplitudes of the resonance peaks in the model are arbitrary owing to the absence of damping. There are also four weakly excited resonances that are apparent in the measurement but not in the FE model.

\section{Modal analysis}

Table 4 lists the natural frequencies predicted by the FE model below $300 \mathrm{~Hz}$ and classifies the modes as those in the plane of the L-beam $(x-y)$ and those that are out of plane $(z)$ and therefore not excited by a vertical base input. The measured resonance peak at $24 \mathrm{~Hz}$ in Fig. 10 (a) is believed to correspond to an out of plane mode at $27.1 \mathrm{~Hz}$ which is excited owing to slight asymmetry of the experimental rig.

Table 4 Natural frequencies of the isolator obtained from FE modal analysis

\begin{tabular}{|c|rc||c|r|}
\hline Order & $\begin{array}{c}\text { Natural frequency } \\
{[\mathrm{Hz}]}\end{array}$ & Order & $\begin{array}{c}\text { Natural frequency } \\
{[\mathrm{Hz}]}\end{array}$ \\
\hline \hline 1 & $1.54 \quad x-y$ & 7 & $64.7 \quad x-y$ \\
\hline 2 & $7.34 \quad x-y$ & 8 & $105.4 \quad x-y$ \\
\hline 3 & $10.4 \quad z$ & 9 & $131.9 \quad x-y$ \\
\hline 4 & 27.1 & $z$ & 10 & $238.4 \quad x-y$ \\
\hline 5 & $37.5 \quad x-y$ & 11 & $265.0 \quad x-y$ \\
\hline 6 & $63.4 \quad z$ & & \\
\hline
\end{tabular}

A high speed camera was used to measure the deflection of the L-shaped beam whilst being subjected to harmonic base excitation at each resonance in turn. Fifty points were measured with a spacing of 10mm, as shown in Fig. 11. Table 5 lists the camera settings for each frequency. It was also necessary to use a higher amplitude of base excitation than selected for the experiment in section 5 for reasons of signal to noise.

The $x$ - $y$ coordinate values were obtained for each of the 50 points on the video image using image analysis software and subsequently transformed to remove their temporal means. Proper orthogonal modes (POM) were identified by application of Proper Orthogonal Decomposition to the measured displacements at each frequency (Feeny and Kappagantu, 1998). Note that POMs are orthogonal to each other, not the mass and stiffness matrices, and so are not in general equivalent to modes. However, they can be expected to resemble the modes of vibration.

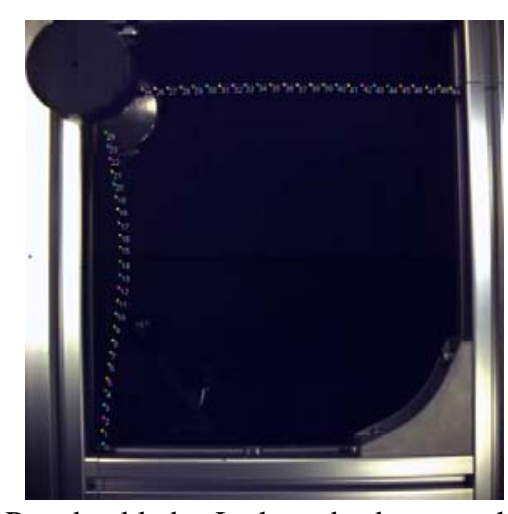

Fig. 11 Post-buckled L-shaped beam showing motion at 50 points obtained from image analysis.

Table 5 Camera settings at each frequency

\begin{tabular}{|c|c|c|c|c|c|}
\hline $\begin{array}{c}\text { Frequency } \\
{[\mathrm{Hz}]}\end{array}$ & 2.0 & 7.2 & 41.0 & 57.0 & 129.1 \\
\hline $\begin{array}{c}\text { Frame rate } \\
\text { [frame/s] }\end{array}$ & 250 & 1000 & 3000 & 1000 \\
\hline $\begin{array}{c}\text { Shutter } \\
\text { speed [s] }\end{array}$ & $1 / 400$ & $1 / 2000$ & $1 / 4000$ & $1 / 2000$ \\
\hline $\begin{array}{c}\text { Recording } \\
\text { time [s] }\end{array}$ & 10.0 & 2.0 & 1.82 & 2.0 \\
\hline
\end{tabular}


Figure 12 shows a comparison of the FE mode shapes with the experimentally obtained POMs. There is reasonable agreement for the first two modes and some significant differences thereafter. It is clear though that higher order modes of the isolator do occur within the frequency range of primary interest.

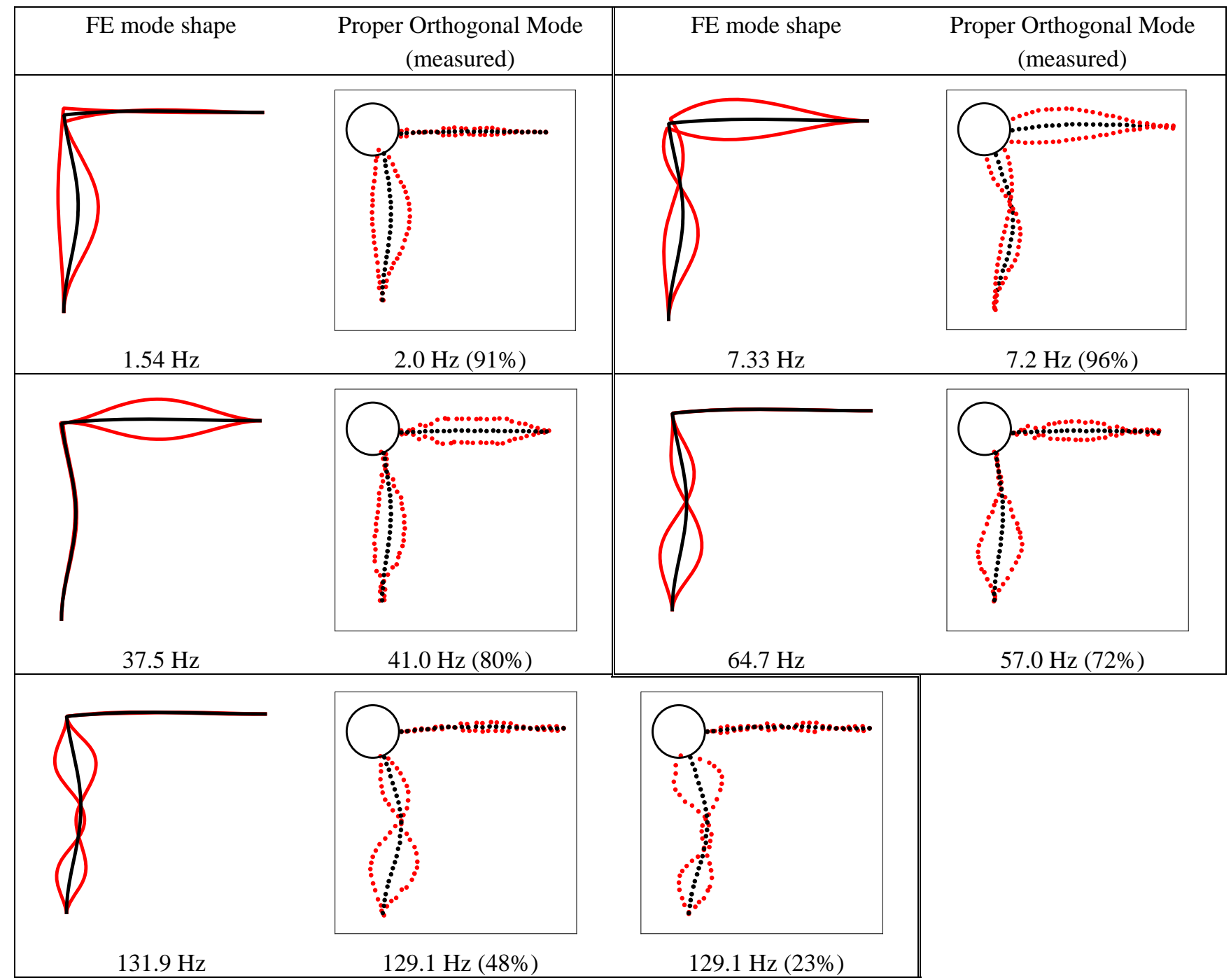

Fig. 12 Comparison of mode shapes from FE analysis and POMs from high speed camera images. The percentage value in parentheses indicates the contribution of that POM to the measured deflection shape at that frequency.

\section{Improvement of the isolator}

Material and geometric properties of the isolator can be adjusted to mitigate against the occurrence and excitation of higher order modes in the frequency range of interest. An example of a modified beam geometry is included here for illustrative purposes. The dimensions of the modified design are listed in Table 6 . In the case considered, the system was arranged to have a static deflection of just $1 \mathrm{~mm}$, i.e. to operate closer to the buckling region. Note that, were the dynamic displacement to exceed this value, then the system could in practice experience a strong hardening stiffness in one direction and possibly switch between one buckled state and the other.

Fig. 13 shows the transmissibility calculated by the FE model. The fundamental frequency of this system is 0.21 $\mathrm{Hz}$ but the isolation region is determined by the second natural frequency at $5.9 \mathrm{~Hz}$ which is strongly excited. This still represents a notable benefit since a comparable linear spring would give rise to a natural frequency of $15.8 \mathrm{~Hz}$. Beyond the second natural frequency the isolation region extends for nearly two decades.

Table 7 lists the natural frequencies up to the 12th order predicted by the FE model and classifies the modes as those in the plane of the L-beam $(x-y)$ and those that are out of plane (z). Figure 14 shows the corresponding mode shapes for the first four in-plane modes. The modification to the isolator has been successful in shifting the second 
mode to 30 times the frequency of the fundamental mode. However, its prominence in the transmissibility needs to be addressed in future studies.

Table 6 Specification of the modified isolator

\begin{tabular}{|c|c|c|}
\hline \multirow{2}{*}{$\begin{array}{c}\text { Vertical } \\
\text { beam }\end{array}$} & Length [mm] & $100 \mathrm{~mm}$ \\
\cline { 2 - 3 } & Thickness [mm] & $0.5 \mathrm{~mm}$ \\
\hline $\begin{array}{c}\text { Horizontal } \\
\text { beam }\end{array}$ & Length [mm] & $100 \mathrm{~mm}$ \\
\hline \multirow{2}{*}{$\begin{array}{c}\text { Isolated } \\
\text { object }\end{array}$} & Thickness [mm] & $0.7 \mathrm{~mm}$ \\
\hline$-\begin{array}{c}\text { Moment of inertia } \\
{\left[\mathrm{kg} \cdot \mathrm{m}^{2}\right]}\end{array}$ & $11.3 \mathrm{~kg}$ \\
\hline Coil spring & $\begin{array}{c}\text { Spring constant } \\
{[\mathrm{N} / \mathrm{m}]}\end{array}$ & $I_{z}=56.50 \times 10^{-3}$ \\
\hline
\end{tabular}

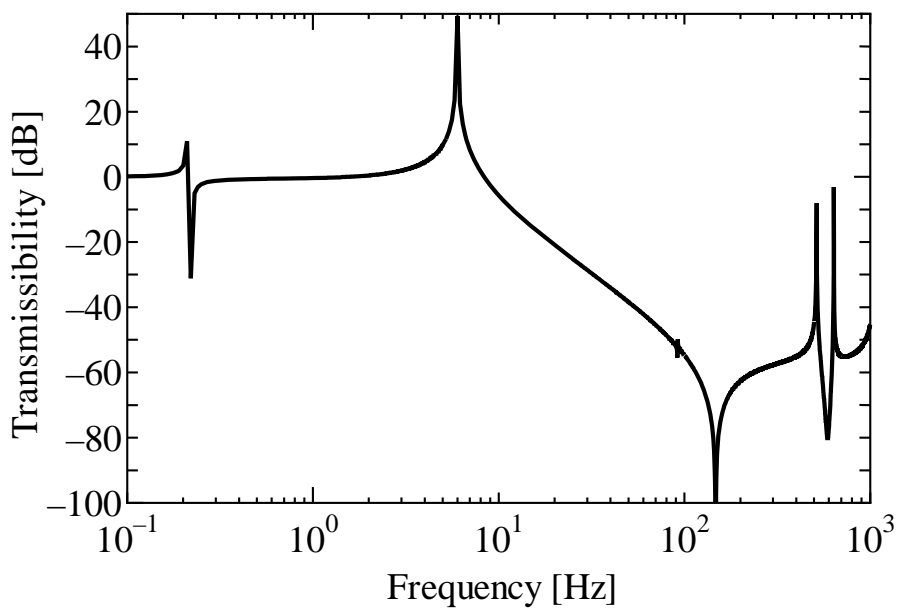

Fig. 13 Transmissibility calculated by the FE model of the modified system specified in table 6 . The fundamental frequency of this system is $0.21 \mathrm{~Hz}$ and the second natural frequency is $5.9 \mathrm{~Hz}$. Beyond the second natural frequency the transmissibility is unaffected by isolator resonances below $500 \mathrm{~Hz}$.

Table 7. Natural frequencies of the modified isolator obtained from FE modal analysis

\begin{tabular}{|c|c|c|c|c|c|}
\hline Order & \multicolumn{2}{|c|}{$\begin{array}{c}\text { Natural frequency } \\
{[\mathrm{Hz}]}\end{array}$} & \multirow{2}{*}{$\begin{array}{c}\text { Order } \\
7 \\
\end{array}$} & \multicolumn{2}{|c|}{$\begin{array}{c}\text { Natural frequency } \\
{[\mathrm{Hz}]}\end{array}$} \\
\hline 1 & 0.21 & $x-y$ & & 515.0 & $x-y$ \\
\hline 2 & 5.99 & $x-y$ & 8 & 637.6 & $x-y$ \\
\hline 3 & 7.26 & $Z$ & 9 & 1050.5 & $x-y$ \\
\hline 4 & 13.4 & $Z$ & 10 & 1366.5 & $x-y$ \\
\hline 5 & 42.1 & $Z$ & 11 & 1473.7 & $Z$ \\
\hline 6 & 91.8 & $x-y$ & 12 & 1595.6 & $Z$ \\
\hline
\end{tabular}




\begin{tabular}{|c||c|c|c|c|}
\hline $\begin{array}{c}\text { Order and } \\
\text { natural frequency }\end{array}$ & 1 & 2 & 6 & 7 \\
& $0.21 \mathrm{~Hz}$ & $5.99 \mathrm{~Hz}$ & $91.8 \mathrm{~Hz}$ & $515.0 \mathrm{~Hz}$ \\
\hline \multirow{2}{*}{ Mode shape } & & & \\
\hline
\end{tabular}

Fig. 14 Mode shapes of the modified isolator obtained from FE modal analysis (first four in-plane modes)

\section{Conclusions and future work}

This paper has investigated the static and dynamic characteristics of a nonlinear vibration isolator based on a post-buckled inverted L-shaped beam. Quasi-static tests have been conducted to measure the negative stiffness in its unstable buckling mode and thereby to identify a suitable coil spring to insert by which a near zero-stiffness system can be realised. The natural frequency was found to be about one-fifth of what would be achieved for an equivalent linear system and slightly amplitude dependent for large dynamic oscillations owing to its predominant softening stiffness. The transmissibility was sought for the linearized system about a chosen equilibrium position in the buckled region. Measured results for a harmonic base input were in reasonable agreement with FE analysis. The potential benefit of the nonlinear mount is apparent but is compromised to some extent by the excitation of higher order modes of the isolator. Experimental and FE modal analyses have been conducted to inform future optimisation of the mount.

\section{Acknowledgement}

This work was supported by JSPS Grant-in-Aid for Young Scientists (B) Number 80343432. The measurement of the beam deflection in section 6 was carried out with a high speed camera at the Instrumentation Center, University of Kitakyushu.

\section{References}

Abolfathi, A., Waters, T.P. and Brennan, M.J., On the performance of a nonlinear vibration isolator consisting of axially loaded curved beams, Recent Advances in Structural Dynamics (2013), Pisa.

Araki, Y., Asai, T., Kimura, K,, Maezawa, K., Masui, T., Nonlinear vibration isolator with adjustable restoring force, Journal of Sound and Vibration, 332(23) (2013), pp. 6063-6077.

Carrella, A., Passive vibration isolators with high-static-low-dynamic-stiffness, PhD thesis (2010), University of Southampton.

Carrella, A., Brennan, M.J., Waters, T.P. and Shin, K., On the design of a high-static-low-dynamic stiffness isolator using linear mechanical springs and magnets, Journal of Sound and Vibration, 315(3) (2008), pp. 712-720.

Feeny, B.F. and Kappagantu, R., On the Physical Interpretation of Proper Orthogonal Modes in Vibrations, Journal of Sound and Vibration, 211(4) (1998), pp. 607-616.

Ibrahim, R. A., Recent Advances in Nonlinear Passive Vibration Isolators, Journal of Sound and Vibration, 314 (2008), pp. 371-452.

Le, T., Ahn, K.K., A vibration isolation system in low frequency excitation region using negative stiffness structure for vehicle seat, Journal of Sound and Vibration, 330(26) (2011), pp. 6311-6335.

Park, S. T. and Luu, T. T., Techniques for Optimizing Parameters of Negative Stiffness, Proc of IMechE, Journal of Mechanical Engineering Science, Vol. 221 (2007), pp. 505-511.

Robertson, W.S.P., Cazzolato, B., Zander, A., Experimental results of a 1D passive magnetic spring approaching quasi-zero stiffness and using active skyhook damping, Proceedings of Acoustics 2013 (2013), Victor Harbor, Australia. 
Shaw, AD, Neild, SA, Wagg, DJ, Weaver, PM and Carrella, A, A nonlinear spring mechanism incorporating a bistable composite plate for vibration isolation, Journal of Sound and Vibration, 332(24) (2013), pp. 6265-6275.

Tsuji, Y., Sasaki, T., Waters, T.P., Fujito, K. and Wang, D., A Nonlinear Vibration Isolator Based on a Post-buckled Inverted L-shaped Beam, The Sixth World Conference on Structural Control and Monitoring (2014), Barcelona.

Winterflood, J., Blair, D. G. and Slagmolen, B., High Performance Vibration Isolation using Springs in Euler Column Buckling Mode, Physics Letters A, Vol. 300 (2002), pp. 122-130.

Xingtian Liu, Xiuchang Huang, Hongxing Hua, On the characteristics of a quasi-zero stiffness isolator using Euler buckled beam as negative stiffness corrector, Journal of Sound and Vibration, 332(14) (2013), pp. 3359-3376. 